\title{
Efectividad decisoria en ambientes culturales diversos y experiencias previas en misiones de paz: Un estudio correlacional con cascos azules argentinos
}

\author{
Pablo Domingo Depaula ${ }^{1}$ y Susana Celeste Azzollini²
}

\begin{abstract}
Artículo
Material original autorizado para la publicación en la revista Psicodebate. Facultad de Ciencias Sociales. Universidad de Palermo.

Recibido 18-02-2016 | Aceptado 01-03-2016
\end{abstract}

\section{Resumen}

Actualmente, algunas investigaciones sobre toma de decisiones señalan que, ante determinadas tareas o contextos específicos, la intuición podría resultar más efectiva que el análisis. Sin embargo, poco se conoce acerca de la efectividad decisoria alcanzada en ambientes culturales diversos que implican la presencia de rasgos culturales diferentes de los propios de la cultura del decisor, sus relaciones con las experiencias previas de trabajo en dichos países huésped, ni sobre la influencia de la edad sobre la primera. Este estudio propone evaluar las relaciones entre la efectividad de las decisiones tomadas en ambientes culturales diversos y la participación previa de los decisores en la cultura anfitriona, y en qué medida su edad se encuentra asociada a la efectividad alcanzada. Para ello, se seleccionó una muestra no probabilística intencional constituida por 130 cascos azules, los que se encontraban recibiendo entrenamiento y capacitación en el Centro Argentino de Entrenamiento Conjunto para Operaciones de Paz (CAECOPAZ), puesto que durante los seis meses posteriores participarían en una misión de paz de Naciones Unidas a desarrollarse en la República de Haití. Los participantes respondieron a una tarea experimental de toma de decisiones (bajo la consigna de hacerlo de modo intuitivo -emocional- o analítico

1 Instituto de Investigaciones en Psicología, Universidad de Buenos Aires (UBA), CONICET. Centro de Investigaciones Sociales y Humanas para la Defensa (CISOHDEF), Instituto Universitario del Ejército (IUE), Universidad de la Defensa Nacional (UNDEF), Buenos Aires, Argentina. Dirección de correo electrónico: lic_pablodepaula@yahoo.com.ar

2 Instituto de Investigaciones en Psicología, Universidad de Buenos Aires (UBA), CONICET. Centro de Investigaciones Sociales y Humanas para la Defensa (CISOHDEF), Instituto Universitario del Ejército (IUE), Universidad de la Defensa Nacional (UNDEF), Buenos Aires, Argentina. 
-racional-) en ambientes de baja o alta incertidumbre cultural, presentada a través de un software específico; posteriormente, respondieron a una encuesta sociodemográfica especificando la cantidad de participaciones en misiones de paz que habían experimentado previamente y otras variables como la edad, el sexo, entre otras. Los resultados evidenciaron una asociación positiva significativa entre la cantidad de participaciones previas en misiones de paz en Haití y la efectividad decisoria alcanzada, sin que la edad de los sujetos se halle asociada a la segunda. Futuros estudios deberían explorar las decisiones efectivas ante el fenómeno de la diversidad cultural en áreas no militares (i.e., empresarial, educativa, sanitaria).

Palabras Clave: efectividad decisoria, experiencias previas, diversidad cultural, misiones de paz.

\section{Effective decision making in diverse cultural environments and previous experiences in peacekeeping missions: A correlation study with argentine peacekeepers}

\section{Abstract}

Currently, some research on decision making point that in certain tasks or contexts intuition may be more effective than analysis. However, little is known about the determining effectiveness achieved in different cultural environments which involve the presence of different cultural traits of the culture of the decision maker, the relationships with previous work experience in these host countries and the influence of age on the first. This study aims to evaluate the relationship between the effectiveness of the decisions taken in different cultural environments and prior involvement of decision makers in the host culture and to what extent its age is associated with the effectiveness reached. To do this, an intentional non-probabilistic sample made up of 130 peacekeepers was selected. They were under training in the Argentine Joint Training Centre for Peacekeeping Operations (CAECOPAZ) prior to their six-month deployment in the UN mission in the Republic of Haiti. Participants responded to an experimental task of decision making (under the banner of doing so intuitive -emotional- or analytical -rational-) in environments of low or high cultural uncertainty, presented through a specific software; subsequently they responded to a demographic survey specifying the number of entries in peacekeeping missions that had previously 
experienced and other variables such as age, sex, and others. The results showed a significant positive association between the number of previous participation in peacekeeping missions in Haiti and the determining effectiveness achieved without the age of the subjects is found associated with the second. Future studies should explore effective decisions to the phenomenon of cultural diversity in nonmilitary areas (i.e., business, education, health).

Keywords: effective decision making, previous experiences, cultural diversity, peacekeeping missions. 
La toma de decisiones presenta un aspecto psicológico determinante de las conductas de elección de los seres humanos en diversos contextos. La información disponible acerca de los hechos sobre los que se va a decidir resulta de suma importancia, puesto que permitiría tomar decisiones con un alto nivel de certeza (e.g., en términos del grado de efectividad que se alcanzará) bajo completa ignorancia o desconocimiento de los resultados, o bien con cierto nivel de riesgo.

La efectividad operativa alcanzada tras la aplicación de modelos decisorios racionales o analíticos recibiría el impacto de factores tales como la presión del tiempo, la disponibilidad y el uso de información inadecuada, y el ritmo rápido de los cambios del ambiente. En contraste, el concepto de la intuición opera integrando componentes cognitivos y afectivos, aseverando la idea de interconexión entre la toma de decisiones analítica e intuitiva (Sinclair \& Ashkanasy, 2005); de ahí que surgen dos alternativas para comprender este constructo: a) la perspectiva basada en la experiencia, donde la intuición es considerada como una forma de expertise cristalizada en hábito (e.g., Campitelli, 2006; Simon, 1955, 1987), de naturaleza no-verbal, cuyos conocimientos, que intervienen en el procesamiento intuitivo, remiten, en mayor medida, a experiencias del pasado (Crossan, Lane \& White, 1999); b) la perspectiva basada en el afecto, que opera de modo pre-verbal, legitimando la importancia de aquellos conocimientos que, sin alcanzar la jerarquía cognitiva funcional y estructural de la expertise, dan lugar a insights decisorios, configurando una nueva estructura cognitiva de patrones de reconocimiento interconectados (Crossan et al., 1999). Más adelante, Sinclair (2010) argumenta que la consideración de ambas perspectivas resulta "artificial", dado que experiencia y afecto representan diferentes facetas de un mismo constructo de naturaleza multidimensional, denotando la discrepancia o falta de consenso teórico y empírico que existe en relación con la unidimensionalidad, bidimensionalidad o multidimensionalidad que caracterizaría a los procesos de toma de decisiones.

\section{La efectividad de la toma de decisiones}

Más allá de la existencia de gran cantidad de estudios que han distinguido modelos analíticos e intuitivos, Aczel, Lukacs, Komlos y Aitken (2011) afirman que los intentos de contrastar los efectos de la intuición y el pensamiento deliberado han sido escasos por dos motivos: por la dificultad para evaluar la bondad o el nivel de eficacia alcanzado al tomar una decisión particular, y por la suposición tradicional de que razonamiento y análisis siempre conducen a la mayor eficacia en materia de resultados.

Algunos autores enfatizan la importancia de comprender cuándo el uso de la intuición sería probablemente exacto o inexacto, sobre todo bajo circunstancias en las 
que la aplicación de decisiones deliberadas o intuitivas puede conducir a resultados divergentes (Salas, Rosen \& Diaz Granados, 2010). Particularmente, cuando la experiencia personal sugiere una opción decisoria diferente de la aplicación de alguna regla ajena al contexto o ambiente de acción, las consecuencias producto de errores del decisor pueden alterar la dinámica de cualquier sistema (Salas et al., 2010). Para estos autores, los ambientes dinámicos e inciertos representan una amenaza que incide en el nivel de equipo y en su orientación, y afectan su rendimiento, los resultados, el manejo de los conflictos, para lo cual el uso de la expertise basada en la intuición sobre escenarios complejos representaría un recurso idóneo.

Del mismo modo que el entrenamiento de la intuición resulta efectivo en tanto permite la adquisición de expertise, la incorporación de factores sociales y culturales característicos en la toma de decisiones (variando de acuerdo con la localización territorial donde se desarrollen las tareas), puede transferirse al entrenamiento a través de la integración de equipos multiculturales, o mediante el uso de la simulación de diversos escenarios culturales de decisión-acción, facilitando el manejo de las intuiciones o emociones (Frank, Curry, Wheaton, Hill \& Abbott., 2011; Salas et al., 2010; Zimmerman, Mueller, Marcon, Daniels \& Vowels, 2011).

En relación con los estudios neurocientíficos realizados en el área, Sinclair (2010) argumenta que la identificación de dos sistemas o estructuras neurales implicadas en el procesamiento de la información encargados de regular la automaticidad y el control (e.g., Lieberman, Jarcho \& Satpute, 2004) no ha considerado debidamente la impronta del nivel de experiencia previa del decisor sobre el despliegue de las operaciones mentales, dependiendo de la primera los resultados alcanzados.

Algunos autores reconocen que tanto la implicancia de los aspectos disposicionales subjetivos, la dependencia contextual de parte del procesamiento intuitivo, como la participación emocional o el rol del afecto en la toma de decisiones y su efectividad, no han sido debidamente investigadas (Cools, 2009; Hodgkinson \& Starbuck, 2008; Sadler-Smith, 2011; Sinclair, 2010; Sinclair \& Ashkanasy, 2005).

Considerando las mencionadas premisas teóricas, Raab y Laborde (2011) seleccionaron una muestra integrada por 54 jóvenes jugadores de hándbol (27 varones y 27 mujeres, con una media de edad de 15.27 años), los cuales presentaban diferentes niveles de expertise vinculados a esta práctica deportiva (sujetos expertos, casi expertos y no expertos), conforme al promedio de años de entrenamiento que había recibido cada grupo. La consigna de la tarea experimental consistía en solicitarle a los jugadores, en posesión del balón, que elijan la primera opción decisoria que viniera a su mente (ya sea, dando un pase a otro jugador de su equipo o disparando al arco contrario) luego de visualizar una serie de fotogramas correspondientes a 15 clips digitales de video proyectados en un lapso de 10 segundos (idénticos para todos los grupos experimentales), los que en determinado 
momento eran "congelados" durante 6 segundos, representando jugadas o posiciones tácticas de ataque. Posteriormente, los jugadores debían generar una mayor cantidad de opciones alternativas de respuesta táctica apropiadas, y elegir el curso de acción que considerasen como la mejor opción decisoria; los participantes fueron evaluados mediante la escala Preference for Intuition and Deliberation (PID, versión alemana; Betsch, 2004) a fin de determinar sus preferencias para la toma de decisiones (intuitivas o deliberadas; e.g., Betsch \& Kunz, 2008; Evans, 2003; Pacini \& Epstein, 1999). Los resultados del estudio anterior indicaron que las mejores y más rápidas decisiones tomadas correspondieron a los jugadores con un mayor nivel de expertise, tanto en relación con las primeras como a las mejores opciones generadas (las que fueron inferiores en número), comparados con los sujetos no expertos; al tiempo que no se hallaron diferencias significativas entre el rendimiento de los grupos de sujetos casi expertos y no expertos. Más adelante, sobre la base de estas conclusiones, Raab (2012) y de Oliveira, Lobinger y Raab (2014) argumentaron que los expertos desarrollan una "caja de herramientas" integrada por heurísticos que operan como guías decisorias.

\section{Algunas notas acerca de la diversidad cultural}

En las últimas décadas, el fenómeno de la globalización ha prolongado intensamente el desarrollo de prácticas profesionales ancladas en el escenario heterogéneo de la diversidad cultural, trascendiendo de modo transversal los límites territoriales y fomentando el pluralismo de valores humanos -sobre todo, en términos de individualismo/colectivismo- (Hofstede, 1980, 2001) y los dominios cognitivos o habilidades culturales específicas (Depaula, 2010; Depaula \& Azzollini, 2012).

Este complejo panorama contextual configura la importancia que revisten los procesos de toma de decisiones en el marco de las misiones de paz desarrolladas bajo el mandato de Naciones Unidas, las que se encuentran atravesadas por una multiplicidad de aspectos culturales. En tal sentido, resulta de importancia capital revisar algunas cuestiones relativas a los ambientes culturales diversos y a las competencias o habilidades de tal índole requeridas para tomar decisiones en el marco de dichos escenarios.

Las capacidades y competencias requeridas para funcionar de forma eficaz en ambientes culturales diversos no han sido debidamente sistematizadas, configurando una brecha crítica en la definición de los recursos socioculturales y psicológicos necesarios para alcanzar el mayor nivel de efectividad y ajuste intercultural (Lin, Chen \& Song, 2012). Algunos autores sostienen que proporcionar a los expatriados información acerca de normas y comportamientos culturalmente apropiados al contexto de trabajo, el apoyo social endogrupal horizontal (entre pares) y vertical 
(supervisión), resulta útil para reducir la incertidumbre de los mismos (e.g., Harrison, Shaffer \& Bhaskar-Shrinivas, 2004). No obstante, la incorporación de este tipo de conocimiento o background cultural acerca del país huésped no basta para asegurar el ajuste intercultural, sino que es necesario que los expatriados presenten cierto nivel de conciencia y reflexión cultural -metacognición- (Ang et al., 2007) evitando el fracaso en su rendimiento (Lin et al., 2012).

En consonancia con lo expuesto arriba, Ross y Thornson (2008) sugieren abordar las competencias culturales (i.e., el ajuste intercultural, la inteligencia cultural y/o la empatía etnocultural) en relación con los niveles de experiencia profesional (e.g., militar). Considerando lo anterior, los citados autores coinciden con la postura de Klein y colaboradores (e.g., Klein, 1998; Ross, Phillips, Klein, \& Cohn, 2005), en lo que atañe directamente a la importancia de la expertise para la toma de decisiones, puesto que, cuanto mayor es el nivel de esta última, menor es la necesidad de cierre cognitivo y, en consecuencia, la adherencia a la estructura funcional y al plan inicial, y la intolerancia a la ambigüedad del ambiente decisorio.

Bajo este panorama contextual, las misiones de paz de Naciones Unidas constituyen un genuino ejemplo de prácticas profesionales desplegadas frente a la diversidad cultural. En tales operaciones, los cascos azules o peacekeepers se desempeñan tomando decisiones en diversas zonas de conflicto internacional. El desarrollo de estas misiones exige cotidianamente el establecimiento de relaciones con nativos o lugareños que integran la población local y con profesionales militares (del Ejército, la Armada y/o la Fuerza Aérea), provenientes de otras Fuerzas de Seguridad (e.g., Gendarmería) o civiles (e.g., traductores de idiomas), pertenecientes a otras organizaciones no gubernamentales o a fuerzas armadas extranjeras, al divergir radicalmente sus rasgos étnicos, religiosos, idiomáticos, etc. (Azzollini, Torres, Depaula, Clotet, \& Nistal, 2010a, 2010b; Torres, Azzollini, Depaula, \& Clotet, 2010).

Depaula, Azzollini y Torres (2012) afirman que frente a tales contextos multiculturales, las comunidades e instituciones de cada país se encuentran inmersas en escenarios globales e inciertos que requieren, de los diversos actores sociales, la posesión de habilidades y conocimientos específicos que les permitan comunicarse y comprender a otras personas que portan valores y tradiciones culturales diferentes, condicionando, en ocasiones, las prácticas del liderazgo intergrupal, sobre todo frente a situaciones de ambigüedad y crisis.

Para sintetizar, los mencionados contextos de acción requieren la adaptación y la toma de decisiones, lo que implica elevados niveles de incertidumbre informativa y ambiental, los que influyen habitualmente sobre los niveles operacionales del componente militar que participa activamente de las tareas desarrolladas en una misión de paz típica. 


\section{Algunos tópicos acerca de la toma de decisiones y su efectividad en relación a la edad}

Una amplia cantidad de evidencias empíricas establece que la edad incide en el rendimiento alcanzado en diversas tareas, especialmente al comparar adultos jóvenes con mayores, ya que estos últimos son menos eficaces en aquellas tareas de evocación que implican procesos de recuperación de memoria episódica. Estas diferencias no son tan claras cuando las pruebas de memoria son de reconocimiento (Azzollini \& Piñeyro, 2010).

En la actualidad, los enfoques neuropsicológicos tratan de hallar una explicación a las diferencias en la efectividad alcanzada en tareas cognitivas vinculadas a la edad, a causa de un deterioro progresivo del lóbulo frontal. No obstante, pese a existir evidencias del déficit en el rendimiento alcanzado en aquellas situaciones que involucran principalmente el uso del ejecutivo central y la memoria de trabajo (funciones que dependen de la activación de la región dorsolateral del lóbulo frontal), aún no se han hallado diferencias ligadas a la edad en gran parte de las tareas que implican la toma de decisiones emocionales y sociales, dominios dependientes del área ventromedial prefrontal (MacPherson, Phillips \& Della Sala, 2002).

Albert y Steinberg (2011) argumentan que los estudios acerca del desarrollo de modelos duales de procesamiento cognitivo (Epstein, 1994) han evidenciado asociaciones entre la edad y el uso progresivo de diferentes clases de heurísticos. Por otro lado, señalan que frente a la resolución de tareas que demandan decisiones vinculadas con contenidos de tipo social, a mayor edad aumenta la aplicación incorrecta del razonamiento sobre el problema a través de información estereotipada (e.g., Markovits \& Dumas, 1999), falacias de la conjunción (Davidson, 1995) y uso del heurístico de representatividad (Jacobs \& Potenza, 1991). Específicamente, durante la adolescencia, en ciertos contextos o ambientes que activan esquemas sociales singulares, el uso de heurísticos parece influir sobre el curso del desarrollo (Albert \& Steinberg, 2011).

Con el objetivo de corroborar experimentalmente si existían diferencias en los modos de procesamiento de la información y el rendimiento alcanzado en la toma de decisiones, en función de la edad de las personas, Azzollini, Piñeyro, Depaula, Castillo y Cosentino (2013) evaluaron el estilo de procesamiento decisorio y la efectividad de las decisiones tomadas por cascos azules de distintas edades frente a las diferentes situaciones en las que se manipuló experimentalmente el grado de incertidumbre cultural propio del ambiente decisorio. Los resultados indicaron que los sujetos con mayor edad presentaron una clara tendencia hacia el uso de procesos predominantemente intuitivos en la toma de decisiones, la cual se 
mantuvo incluso después de controlar los efectos de la experiencia. También se encontró una pérdida marginalmente significativa de la efectividad decisoria en los sujetos de mayor edad, pero al controlar la variable experiencia (participación en misiones de paz), las diferencias en la efectividad tendieron a desaparecer.

Con estos resultados, Azzollini et al. (2013) afirman que si bien se requiere de un mayor número de evidencias científicas, la tendencia natural de nuestro sistema cognitivo hacia el compactamiento de información (mediante el uso de heurísticos), generaría un ahorro de recursos de la memoria de trabajo, pudiendo incrementarse como consecuencia de la maduración biológica, independientemente de la experiencia. Estos autores señalan que si bien los sujetos más jóvenes (entre 18 y 24 años de edad) fueron más efectivos en sus decisiones, análisis posteriores en los que se controló su experiencia previa no arrojaron diferencias estadísticamente significativas, por lo que no existirían diferencias en la efectividad decisoria determinada por la edad, sobre todo en situaciones sociales y emocionales (e.g., MacPherson et al., 2002).

Considerando el problema de investigación planteado, el presente trabajo tiene como objetivo evaluar si existen relaciones entre la efectividad de las decisiones tomadas en ambientes culturales diversos y la participación previa de los cascos azules en misiones de paz en Haití, y en qué medida su edad se encuentra asociada a su efectividad, a fin de testear la hipótesis relativa a que los cascos azules que han participado previamente en misiones de paz en Haití resultan más efectivos en sus decisiones, sin que su edad interactúe en dicha relación.

\section{Método}

\section{Diseño}

Estudio correlacional, con diseño experimental, de corte transversal. Las variables principales son la efectividad decisoria, el nivel de experiencias previas de participación en misiones de paz en la República de Haití y la edad de los decisores.

\section{Participantes}

Se seleccionó una muestra no probabilística intencional constituida por 130 cascos azules, los que al momento de realizar el presente estudio se hallaban recibiendo entrenamiento y capacitación en el Centro Argentino de Entrenamiento Conjunto para Operaciones de Paz (CAECOPAZ), dado que participarían como voluntarios en la denominada Misión de Estabilización de las Naciones Unidas en Haití (MINUSTAH) durante los seis meses posteriores; su edad promedio era de 29.04 años $(D E=5.13$ años $)$, todos de sexo masculino. 


\section{Materiales y procedimiento}

En una primera fase del estudio, los participantes recibieron tratamientos experimentales aplicados a través del desarrollo de un programa informático denominado Software Interactivo de Toma de Decisiones (SITDE 1.0; Depaula et al., 2012), a los fines de evaluar la efectividad decisoria de los cascos azules desplegada en ambientes culturales diversos (dotados -a través de la mencionada herramienta informática- de cierto nivel de realismo situacional propio de la región de Haití, en lo que atañe a sus rasgos o pautas culturales características).

La operacionalización de la variable independiente representada por el tipo de decisiones tomadas (intuitivas vs. analíticas) durante la tarea experimental propuesta, estuvo dada por la manipulación del proceso decisorio mediante la aplicación de una consigna decisoria intuitiva o emocional ("Usted debe basar su decisión en lo que sienta emocionalmente") o de una consigna decisoria deliberada o racional ("Usted debe basar su decisión en lo que piense racionalmente"), conforme al modelo teórico propuesto por Betsch (2004) y Betsch y Kunz (2008). A partir de esta consigna experimental, los participantes (asignados al azar a alguna de las dos consignas decisorias -consigna "emocional": grupos experimentales A y B; consigna "racional": grupos experimentales C y D) debían responder a una tarea en la que se les presentaban seis situaciones experimentales (dos de prueba y cuatro de evaluación; véase Figura 1), posibles y cotidianas con las que un casco azul puede enfrentarse en el contexto de una misión de paz desplegada en Haití. Ante ambas consignas decisorias y ante la totalidad de las situaciones, los sujetos se encontraban en igualdad de condiciones o estados de situación operativa (típico de determinadas tareas de "patrullaje" de Naciones Unidas): el peacekeeper era parte de una patrulla, no contaba con el apoyo de la Policía Nacional de Haití (PNH, debiendo asumir dicho rol), y no disponía de medios de comunicación (e.g., radio, celular) que le permitieran establecer contacto con el centro de operaciones de Naciones Unidas o con la Unidad o Fuerza de Tarea que integraba en dicho momento hipotético (escenario simulado).

Por otro lado, la operacionalización de la variable independiente representada por el nivel de saturación o incertidumbre cultural (baja vs. alta) estuvo dada por la elaboración de las seis escenas mencionadas arriba, de las cuales las escenas de prueba eran idénticas para la totalidad de la muestra, y la posterior serie de cuatro videos variaba conforme a la aparición o no de patrones propios de la cultura haitiana. 


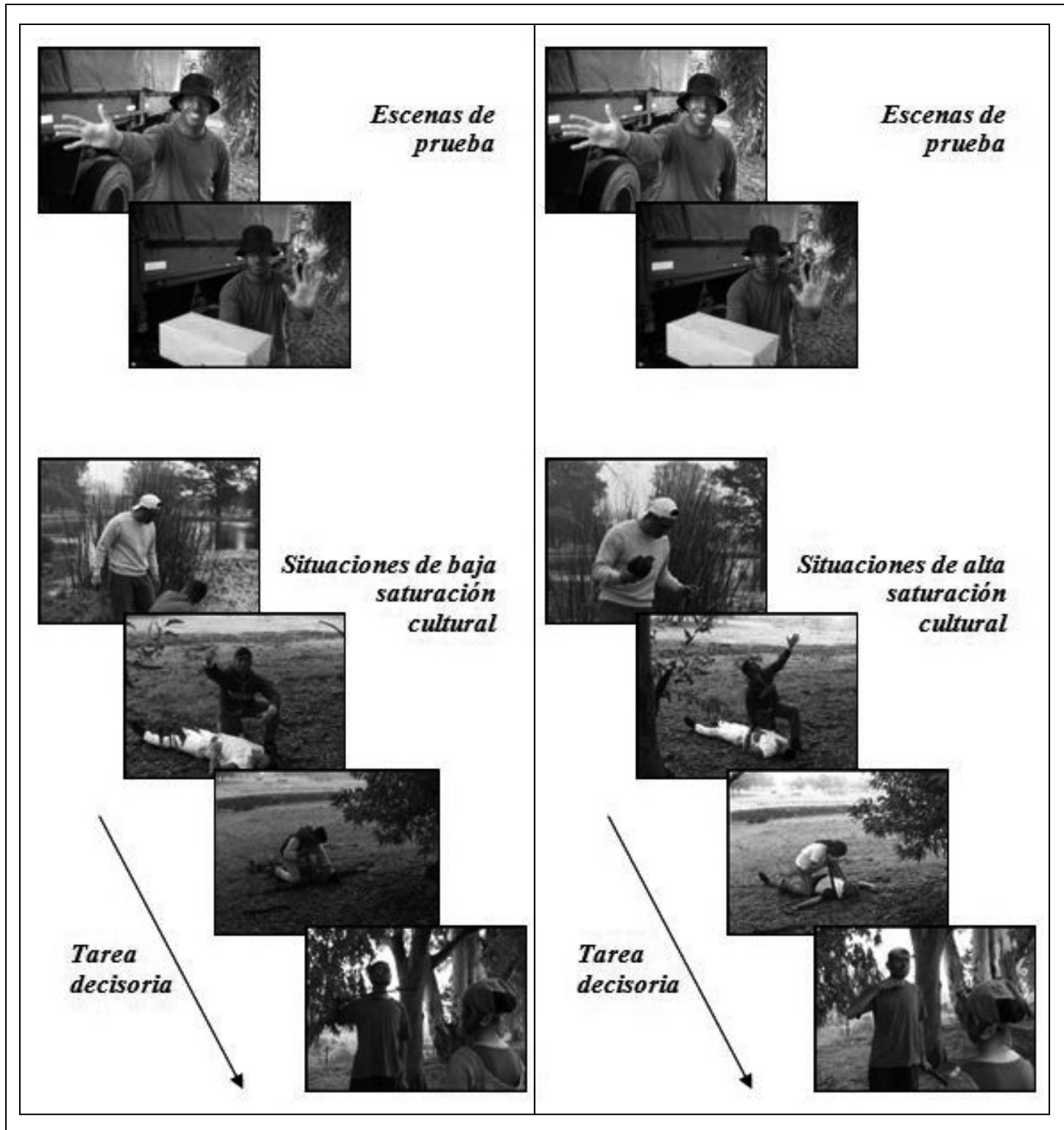

Figura 1. Esquema general del procedimiento experimental de imágenes dinámicas o videos aplicado a través del SITDE 1.0 (situaciones o escenas con diferentes niveles de saturación cultural). Las escenas ubicadas del lado izquierdo de la figura integraron los grupos experimentales B y D (situaciones de baja saturación cultural); las escenas situadas del lado derecho de la figura integraron los grupos experimentales A y C (situaciones de alta saturación cultural). 
Tabla 1.

Tabla de distribución de los sujetos a las condiciones experimentales

\begin{tabular}{cccc}
\hline \multicolumn{5}{c}{ Consigna } \\
\hline \multicolumn{3}{c}{ EMOCIONAL } & \multicolumn{2}{c}{ RACIONAL } \\
\hline Saturación Cultural & Saturación Cultural & Saturación Cultural & Saturación Cultural \\
ALTA & BAJA & ALTA & BAJA \\
(A) & (B) & (C) & (D) \\
32 & 31 & 33 & 34 \\
\hline \multicolumn{5}{c}{} \\
63 & & & 67 \\
\hline
\end{tabular}

Total: 130 sujetos

El nivel de alta saturación cultural (incertidumbre) operacionalizado en los grupos experimentales A y $\mathrm{C}$, estuvo definido mediante elementos típicos de la cultura haitiana seleccionados de acuerdo con la información obtenida precedentemente de la realización de focus groups de los que participaron cascos azules con y sin experiencia en este tipo de misiones de paz (Azzollini, Depaula, \& Torres, 2013), en entrevistas en profundidad realizadas al representante de Comunicación y Cultura de la Embajada de Haití en Argentina, quien facilitara material bibliográfico de interés para el diseño (e.g., Hurbon, 1993), entre otros datos, experiencias y lecciones aprendidas suministradas en las sucesivas reuniones mantenidas con los miembros de la Plana Mayor (jueces expertos) de los contingentes desplegados en la MINUSTAH, los que evaluaron precedentemente el contenido de las escenas que configuraron la versión definitiva del software.

La validez de contenido de las situaciones (en términos de la efectividad decisoria- intervención vs. no intervención-, definida por el tipo de saturación cultural de cada escena), se determinó mediante el juicio emitido por 2 expertos (con rango de Oficiales de las Fuerzas Armadas Argentinas -FF.AA-), dotados de un alto nivel de experiencia en la MINUSTAH (Kappa $=.75 ; p=.028$ ).

En lo que respecta a la valoración de la variable dependiente efectividad decisoria, se consideraron efectivas aquellas decisiones que implicaban la "intervención" (realizar cualquier acción relacionada con la ocurrencia de la escena observada) sobre situaciones de baja saturación cultural (grupos B y D), y aquellas decisiones que implicaban la "no intervención" (no involucrarse en la escena) sobre las situaciones de alta incertidumbre cultural (grupos A y C), conforme a la evaluación consensuada de los miembros de la Plana Mayor del contingente próximo a desplegarse en la MINUSTAH, que participaría del experimento. Nuevamente, y en todos los casos, las escenas de alta saturación cultural eran análogas a las de baja saturación cultural.

Las decisiones efectivas (conforme a la intervención o no intervención esperada 
según el grupo experimental que integrara cada participante) fueron calificadas con el valor de "1" por cada escena o situación decisoria, y las decisiones inefectivas fueron calificadas con el valor de "0" por cada escena o situación decisoria. La efectividad decisoria total alcanzada en la serie de 4 escenas representó un valor absoluto de 4 puntos (máxima efectividad) si se había decidido "intervenir" en la totalidad de las escenas correspondientes a los grupos B y D -baja saturación cultural- o si se había decidido "no intervenir" en la totalidad de las escenas de los grupos A y C -alta saturación cultural-; mientras que la efectividad decisoria total alcanzada en la serie de 4 escenas representó un valor absoluto de 0 puntos (mínima efectividad) si se había decidido "intervenir" en la totalidad de las escenas correspondientes a los grupos A y C -alta saturación cultural- o si se había decidido "no intervenir" en la totalidad de las escenas de los grupos B y D -baja saturación cultural-.

Por otro lado, el nivel de experiencias previas de participación en misiones de paz desarrolladas en la región de Haití fue evaluado con auxilio de un ítem específico que integraba una encuesta de datos socio-demográficos, el cual indagaba dicha variable a través del siguiente enunciado ¿Ha participado de una misión de paz anteriormente?, por lo que los sujetos encuestados debían determinar la cantidad de participaciones experimentadas en las misiones de paz en las que hayan participado bajo el rol de casco azul de Naciones Unidas (i.e., Haití, Chipre, etc.), e informar el año/s en que participaron y el rango jerárquico y rol que cumplieron durante el desarrollo de la misión/es respectiva/s; la cantidad de participaciones en la MINUSTAH fue tipificada numéricamente, asignando valores enteros (valor 0 , cuando se refería a ninguna participación, o bien valor 1, valor 2, valor 3 , según correspondiera para cada caso). Por otro lado, la encuesta mencionada recopilaba datos adicionales tales como la edad (medida en años), el sexo, el estado civil, el lugar de nacimiento y residencia, el nivel de estudios alcanzados por los participantes, entre otra información de menor importancia para el presente estudio.

A los fines de analizar otras hipótesis no contempladas en el presente estudio, el SITDE 1.0 permitió evaluar otras variables tales como el nivel de inteligencia cultural de los participantes, los tiempos que tomó cada una de sus decisiones, y las memorias o recuerdos activados durante los procesos decisorios (juicios de saber o de recordar; e.g., Tulving, 1972, 1983).

Con el consentimiento de las autoridades del CAECOPAZ, los sujetos fueron evaluados -según el grupo experimental A, B, C o D- en un laboratorio de informática e idiomas provisto de recursos tecnológicos consistentes en el hardware requerido de acuerdo con las características de la muestra y el manejo óptimo del SITDE 1.0 (i.e., veinte ordenadores dispuestos de monitores LCD de 17 pulgadas y auriculares) mediante los cuales se presentaron los tratamientos experimentales con auxilio del software. De antemano, se aclaró a los sujetos 
experimentales que la prueba era de carácter anónimo y que ellos podrían renunciar a la continuidad de su participación durante cualquier momento del experimento. Además, éstos firmaron el consentimiento informado correspondiente.

Luego de responder a la tarea experimental, los sujetos eran trasladados a un aula contigua al laboratorio de informática e idiomas, donde respondieron la encuesta socio-demográfica mencionada en la sección anterior mediante un protocolo dispuesto de un formato de lápiz y papel, cuyo tiempo para completarlos no demoraba más de 15 minutos aproximadamente.

\section{Análisis de los datos}

La información recopilada de los tratamientos experimentales aplicados a través del SITDE 1.0 y de la administración de los instrumentos de lápiz y papel fue ingresada en una matriz de datos confeccionada en el software SPSS 22.0 (versión castellana), utilizando dicho paquete estadístico para realizar los análisis que se presentan a continuación.

\section{Resultados}

En primer lugar, para verificar si las muestras respondían a una distribución normal, se llevó a cabo el Test de Kolmogorov-Smirnov indicando que las variables no respondían a ese tipo de distribución $(p<.05)$. En consecuencia, los análisis estadísticos realizados fueron no paramétricos.

Acto seguido, explorando la existencia de asociaciones entre la efectividad decisoria de los sujetos frente a situaciones típicas de la cultura haitiana (enmarcando diferentes niveles de incertidumbre cultural) y el hecho de haber participado previamente en misiones de paz desplegadas en Haití, se realizó la prueba de correlación de Rho de Spearman. Ésta incluía también la edad de los cascos azules, dado que dicha variable podría influir sobre su efectividad (por cuestiones de expertise o bien por ser la variable que realmente influenciara sobre el rendimiento al quedar solapada por la experiencia). Los resultados indicaron que existe una asociación positiva significativa entre la cantidad de participaciones en la MINUSTAH y la efectividad decisoria ( $\rho=$ $.212, p<.05)$, indicando que a mayor número de participaciones en misiones de paz desplegadas en Haití, más efectivas resultan las decisiones tomadas por los cascos azules, sin que la edad de los mismos se halle asociada $(\rho=.069, p=.438)$. Asimismo, la edad se relaciona significativamente de forma positiva con la cantidad de veces que se haya participado en una misión de paz de tal índole $(\rho=.450, p=.000)$, por una simple cuestión de cronología, esperable en los ámbitos militares en alusión al desarrollo de la jerarquía (rangos) y el desempeño, entre otros factores. Con estos resultados, se confirma la hipótesis planteada originalmente. 
Tabla 2.

Relaciones entre la efectividad, la participación previa en misiones de paz en Haití y la edad

\begin{tabular}{|c|c|c|c|c|}
\hline & & & $\begin{array}{l}\text { Cantidad de } \\
\text { veces que } \\
\text { participó en } \\
\text { la MINUSTAH }\end{array}$ & Efectividad \\
\hline \multirow{6}{*}{$\begin{array}{c}\text { Rho de } \\
\text { Spearman }\end{array}$} & \multirow{3}{*}{ Edad } & Coeficiente de correlación & $.450^{\star \star}$ & .069 \\
\hline & & Sig. (bilateral) & .000 & .438 \\
\hline & & $\mathrm{N}$ & 130 & 130 \\
\hline & \multirow{3}{*}{$\begin{array}{l}\text { Cantidad de ve- } \\
\text { ces que participó } \\
\text { en la MINUSTAH }\end{array}$} & Coeficiente de correlación & 1.000 & $.212^{*}$ \\
\hline & & Sig. (bilateral) & . & .016 \\
\hline & & $\mathrm{N}$ & 130 & 130 \\
\hline
\end{tabular}

\section{Comentarios}

Como antecedente significativo de las conclusiones a las que se arribó en el presente estudio acerca de la efectividad de la toma de decisiones, un estudio realizado por Depaula (2016) a través del cual se analizó una muestra de cascos azules voluntarios entrenados para participar de una misión de paz en Haití, en donde se evaluó la toma de decisiones mediante el SITDE 1.0 (Depaula et al., 2012), comprobó que frente a situaciones de mayor incertidumbre cultural, el despliegue de procesos decisorios intuitivos resulta más efectivo, acompañado de la activación de recuerdos semánticos y de menores registros de tiempo decisorio, facilitando el accionar correcto de los cascos azules y evitando el advenimiento de problemas con los lugareños haitianos. La toma de decisiones de tipo intuitiva (emocional) se asocia a la percepción, procesamiento y reconocimiento rápido de estímulos ambientales dotados de una alta saturación cultural. Tales hallazgos coinciden con los resultados de otras investigaciones en dominios deportivos, en las que se realizaron experimentos con auxilio de alta tecnología informática videos en formato visual tridimensional, lentes especiales, $i$ Pod, etc.- (Laborde \& Raab, 2013), mostrando que la intuición, entendida como una forma de emoción, opera, ante determinadas tareas y contextos, como una modalidad decisoria rápida que supera al análisis deliberado, sin dar lugar a demasiados errores sistemáticos.

Los resultados del presente estudio muestran entonces que la participación de los cascos azules en misiones de paz desplegadas en Haití, y su relación con la efectividad 
decisoria, ha permitido observar la manera en que la acumulación de experiencias previas se asocia con la posibilidad de tomar decisiones de forma correcta, lo que se alinea con las ideas de diversos autores (e.g., Dreyfus \& Dreyfus, 2005). Y, coincidiendo con los resultados del estudio anterior realizado por Azzollini et al. (2013), se ha observado que la edad de los decisores no influye sobre su efectividad, pero sí se asocia con las experiencias previas por cuestiones de cronología.

Ahora bien, retomando la importancia de la efectividad de la intuición frente a ambientes culturales diversos, y considerando los hallazgos de Depaula (2016) mencionados en el párrafo anterior, el desarrollo de la experiencia de los cascos azules conduciría a reducir el nivel de confianza y/o aplicación de procedimientos y reglas, y a que incorporen nueva información, perciban situaciones de forma holística, reaccionando con mayor flexibilidad (Fadde, 2009; Ross et al., 2005; Zimmerman et al., 2011). Así, los cascos azules involucrarían datos y conocimientos previos en sus decisiones, relacionándolos de modo global (e.g., Betsch \& Glöckner, 2010) con situaciones en las que detecten ciertas señales o patrones contextuales particulares (Frank et al., 2011).

Según Kahneman y Klein (2009) y Lipshitz, Klein, Orasanu y Salas (2001), el nivel de expertise del decisor lo conduce al uso adecuado del escaso tiempo del que dispone para tomar determinadas decisiones, testeando, criticando y mejorando las opciones decisorias iniciales, en lugar de gastarlo en la generación y contrastación de múltiples opciones (Phillips, Klein \& Winston, 2009). La expertise intuitiva es más confiable en ambientes altamente válidos, es decir donde existe una relación estable entre señales objetivamente identificables y eventos subsecuentes, o entre señales y resultados de posibles acciones. La evaluación correcta de esta validez ambiental dependerá de la "historia de aprendizaje de las reglas de ese entorno" (Kahneman \& Klein, 2009; p. 522), excepto en los denominados ambientes "malvados" o engañosos (Hogarth, 2001, como se citó en Kahneman \& Klein, 2009), circunstancias donde se dificulta el proceso de validación ambiental.

Moxley, Ericsson, Charness y Krampe (2012) destacan el gran número de autores que coinciden con la idea de que los heurísticos e intuiciones conducen mejor y más satisfactoriamente las decisiones cotidianas, incluso las tomadas por expertos (e.g., Dreyfus \& Dreyfus, 2005; Gigerenzer, 2008; Klein, 1998, 2008), al argumentar que una extensa experiencia profesional generalmente no asegura las mejores decisiones, ni aun tampoco son claras las conexiones entre los procesos analíticos e intuitivos asociados a la toma de decisiones expertas. En tal sentido, Moxley et al. (2012) consideran que los procesos cognitivos que despliegan los decisores (e.g., los ajedrecistas), frecuentemente no pueden describirse como simples procesamientos intuitivos o deliberados, sino que demandan la consideración de la intuición sobre la base de teorías afines de mayor complejidad conceptual. 
Abordando los procesos analíticos o prácticas deliberadas, Macnamara, Hambrick y Oswald (2014) realizaron un meta-análisis considerando la relación entre este tipo de prácticas mencionadas y el rendimiento alcanzado en dominios tales como la música, los juegos, los deportes, la educación y las profesiones, en el que hallaron que el uso de la deliberación explicaba el 12\% de la varianza total en el rendimiento. Con estos resultados, los autores sugieren abordar otros dominios o habilidades consideradas potenciales predictoras del rendimiento alcanzado en las prácticas deliberadas, al tiempo que destacan la necesidad de investigar de qué manera y bajo qué tareas y factores situacionales (i.e., la previsibilidad del entorno o ambiente de la tarea) podrían operar moderando el impacto de la deliberación y de otros factores o diferencias individuales que representen significativos predictores del rendimiento en dominios específicos. Así, consideran necesario "desarrollar y probar teorías falsables de la expertise que incluyan tantos conceptos relevantes como sea posible” (Hambrick, Altmann, Oswald, Meinz, \& Gobet, 2014; p. 2).

Vale mencionar algunos estudios en los que altos niveles de experiencia previa, bajo la forma de expertise, no aseguraron el despliegue de las mejores o más efectivas decisiones. Goldstein y Gigerenzer (2009) y Gigerenzer (2008) analizaron la heurística de reconocimiento considerando una investigación en la que una muestra de sujetos con diferentes niveles de conocimientos afines realizaron predicciones de los resultados obtenidos en los partidos de tenis masculino de Wimbledon 2003 (Serwe \& Frings, 2006). En efecto, los sujetos que participaron de la investigación pertenecían a diferentes asociaciones de tenistas profesionales propias de la clasificación oficial y, por el contrario, participaron personas profanas en la materia y aficionados. Los resultados de este estudio indicaron que los legos en la materia y los aficionados adivinaron mayor cantidad de resultados que los miembros de las clasificaciones tenísticas oficiales y que los expertos en Wimbledon.

Al parecer, el despliegue de los procesos decisorios intuitivos o analíticos y su efectividad dependería de dominios identificables y dables de ser activados frente a tareas, estímulos o ambientes específicos (e.g., Laborde, Dosseville, \& Scelles, 2010).

La relevancia que alcanza el tipo de ambiente decisorio (cultural, para el caso) en el presente estudio, en relación con su papel crucial al momento de comprender la conducta de los decisores, coincide con la opinión general de varios autores (e.g., Gibson, 1979; Gigerenzer, Todd, \& the ABC Research Group, 1999; Hammond, 2010; Marr, 1982; McKenzie, 2009; Simon, 1955). Específicamente, vale considerar el "modelo de lentes" (the lens model) de Brunswik (1952; Hammond, 1955) como una eventual alternativa abordable en futuros estudios, ya que dicha teoría funcionalista se orienta a la exploración del ambiente relativo a tareas decisorias complejas, enfatizando en los elementos contextuales que dan lugar a juicios sociales, y evaluando las interrelaciones entre el organismo y el ambiente 
(en el sentido darwiniano del término). Particularmente, este modelo ecológico (en consonancia con los aportes de Gigerenzer, 2008, 2009; Gigerenzer \& Kurz, 2001; Gigerenzer, et al., 1999; Goldstein \& Gigerenzer, 2002, 2009) prioriza la identificación perceptiva (proximal) de rasgos o señales del ambiente decisorio (juicios subjetivos) correlacionados probabilísticamente con los objetos distales (valores o variables de criterio ambiental), por lo que son típicamente "ambiguas" las primeras (Goldstein, 2009), legitimando la cuantificación del procesamiento de la información en lo que respecta ciertos dominios específicos o ambientes complejos (Horrey, Lesch, Mitsopoulos-Rubens \& Lee, 2015).

Analizando las aplicaciones actuales del funcionalismo probabilístico propuesto por Brunswik (1952) y el modelo de racionalidad ecológica que propone Gigerenzer (2008, 2009; Goldstein \& Gigerenzer, 2002, 2009), Goldstein (2009) sugiere una agenda de investigación basada en un modelo de tipo adaptativo, enfocándose en: 1) los heurísticos que son rápidos y frugales; 2) los ambientes en los cuales la aplicación de tales heurísticos resulta en un rendimiento óptimo (efectividad decisoria); 3) el estudio de meta-heurísticos, a través de los cuales las personas eligen deliberadamente los heurísticos a aplicar; 4) la investigación acerca de si las personas actualmente utilizan heurísticos y meta-heurísticos en ambientes y tareas particulares o específicas.

En la misma línea, recientemente algunos autores (e.g., Hammond, 2015) apoyándose en las ideas de Brunswik (1952) sugieren estudiar la intuición a través de diseños que denominan "representativos" en lugar de diseños sistemáticos, ya que los primeros representan los ambientes y estímulos naturales donde las personas toman decisiones cotidianamente y sobre los que "su cognición se ha adaptado" (Hoffrage \& Marewski, 2015; p. 155).

Desde el punto de vista del diseño metodológico, es necesario mencionar una limitación del presente estudio relacionada específicamente con las consignas experimentales asignadas para el despliegue de decisiones de tipo analítico (racional) o intuitivo (emocional), ya que podría ocurrir que los participantes respondan mediante respuestas basadas en heurísticos de disponibilidad, sesgos de emparejamiento, marcadores somáticos, y mediante otras fuentes de información tanto cognitivas como no cognitivas. Al respecto, Evans y Over (1996) enfatizan la notable discrepancia que se registra entre la consigna experimental y los criterios empleados por los participantes al momento de responder a tareas bien definidas, como ocurre con la tarea de selección de Wason $(1968,1969)$ o "problema de las cuatro tarjetas".

Asimismo, las alternativas decisorias de "intervenir" o "no intervenir" ante las situaciones planteadas en la serie o set de videos experimentales aplicados (de baja o alta saturación cultural, según la asignación a los grupos respectivos), podrían corresponder a una tarea de "inhibición binaria" antes que a una decisión conducida 
por la manipulación del criterio emocional o racional, lo cual representaría una limitación del presente estudio. Sin embargo, en general la toma de decisiones (ya sea de naturaleza analítica o intuitiva, en mayor medida) implica siempre la inhibición (más o menos consciente o deliberada) de una o varias alternativas decisorias, en tanto el decisor debe elegir un curso de acción posible. En este sentido, la elección de no intervenir ante situaciones de alta saturación de elementos culturales implicaba en este estudio una decisión "efectiva", y la comprensión de algún aspecto de la cultura haitiana implicaría una conducta culturalmente inteligente y, en efecto, adaptativa al ambiente o contexto (Depaula, 2016), constituyendo más que una inhibición de la acción, un comportamiento prudencial o de respeto cultural, para el caso.

Con todo, futuros estudios deberían abordar con mayor énfasis las diferencias en los procesamientos intuitivos o analíticos y la concomitante toma de decisiones efectivas conforme a la realización de tareas en ambientes culturales diversos en diferentes ámbitos ajenos al desempeño militar (i.e., empresarial, educativo, sanitario, etc.).

\section{Referencias}

Aczel, B., Lukacs, B., Komlos, J. \& Aitken, M. R. F. (2011). Unconscious intuition or conscious analysis? Critical questions for the Deliberation-WhithoutAttention paradigm. Judgment and Decision Making, 4(6), 351-358. doi: 101017/jdm101017

Albert, D. \& Steinberg, L. (2011). Judgment and Decision Making in Adolescence. Journal of Research on Adolescence, 21(1), 211-224. doi: 10.1111/j.15327795.2010.00724.x

Ang, S., Van Dyne, L., Koh, C., Ng, K. Y., Templer, K. J., Tay, C., ... \& Chandrasekar, N. A. (2007). Cultural Intelligence: Its measurements and effects on cultural judgments and decision making, cultural adaptation and task performance. Management and Organization Review, 3(3), 335-371. doi: 10.1111/j.1740-8784.2007.00082.x

Azzollini, S., Depaula, P. D. \& Torres, J. A. (2013). Incertidumbre ambiental, operacional y toma de decisiones en misiones de paz: un abordaje cualitativo. Revista Peruana de Psicología y Trabajo Social, 2(2), 47-60.

Azzollini, S. \& Piñeyro, D. (2010) Incidencias de las expectativas en la memoria episódico-semántica. Investigaciones en Psicología. 15, 2, 49-68.

Azzollini, S., Piñeyro, D., Depaula, P., Castillo, S. \& Cosentino, A. (2013). Cambios de la modalidad decisoria predominante en función de la edad: 
Su relación con la efectividad de las decisiones tomadas por voluntarios en misiones de paz. Anuario de Investigaciones, 19(2), 265-271.

Azzollini, S., Torres, A., Depaula, P., Clotet, C. \& Nistal, M. (2010a, Diciembre). La tensión entre la adaptación y la extrañeza cultural: Los significados atribuidos por integrantes de misiones de paz a los hábitos y costumbres de los lugareños. Trabajo presentado en las VI Jornadas de Sociología "Debates y perspectivas sobre Argentina y América Latina en el marco del Bicentenario. Reflexiones desde las Ciencias Sociales", organizadas por la Universidad Nacional de La Plata, La Plata, Buenos Aires.

Azzollini, S., Torres, A., Depaula, P., Clotet, C. \& Nistal, M. (2010b, Agosto). Los significados atribuidos a las situaciones de incertidumbre por militares integrantes de una misión de paz en Haití. Trabajo presentado en las VI Jornadas de Etnografía y Métodos Cualitativos, organizadas por el Instituto de Desarrollo Económico y Social, y por el Centro de Antropología Social, Ciudad Autónoma de Buenos Aires, Buenos Aires.

Betsch, C. (2004). Präferenz für intuition und deliberation. Inventar zur erfassung von affekt- und kognitionsbasiertem entscheiden [Preference for intuition and deliberation (PID): An inventory for assessing affect- and cognitionbased decision-making]. Zeitschrift für Differentielle und Diagnostische Psychologie, 25, 179-197. doi: 10.1024/0170-1789.25.4.179

Betsch, C. \& Kunz, J. J. (2008). Individual Strategy Preferences and Decisional Fit. Journal of Behavioral Decision Making, 21, 532-555. doi: 10.1002/bdm.600

Betsch, T. \& Glöckner, A. (2010). Intuition in Judgment and Decision Making: Extensive Thinking Without Effort. Psychological Inquiry, 21(4), 279-294. doi: 10.1080/1047840X.2010.517737

Brunswik, E. (1952). The conceptual framework of psychology. Chicago, IL: University of Chicago Press.

Campitelli, G. (2006). Pensamiento organizado: Una psicotecnología para ser feliz, eficiente y colaborar inteligentemente. Buenos Aires: Dunken.

Cools, E. (2009). A reflection on the future of the cognitive style field: a proposed research agenda. Reflective Education, 5(2), 19-34.

Crossan, M. M., Lane, H. W. \& White, R. E. (1999). An Organizational Learning Framework: From Intuition to Institution. Academy of Management Review, 24(3), 522-537. 
Davidson, D. (1995). The representativeness heuristic and the conjunction fallacy effect in children's decision making. Merrill-Palmer Quarterly, 41, 328-346.

de Oliveira, R. F., Lobinger, B. H. \& Raab, M. (2014). An adaptive tool- box approach to the route to expertise in sport. Frontiers in Psychology, 5, 709. doi: 10.3389/fpsyg.2014.00709

Depaula, P. D. (2010). Integración de predictores globales del rendimiento en estudiantes de un instituto militar. Desarrollos y aplicaciones de inteligencia cultural para la defensa. Tesis de Maestría en Psicología Organizacional no publicada, Universidad Abierta Interamericana, Buenos Aires, Argentina.

Depaula, P. D. (2016). Toma de decisiones en ambientes culturales diversos. Tesis Doctoral no publicada, Universidad de Buenos Aires, Argentina.

Depaula, P. D. \& Azzollini, S. C. (2012). Inteligencia cultural, valores y motivación para el aprendizaje en estudiantes militares argentinos. Revista de Psicología, 30(1), 75-102.

Depaula, P. D., Azzollini, S. C. \& Torres, J. A. (2012). Aspectos diferenciales de la inteligencia cultural. Un abordaje en estudiantes militares argentinos. Boletín de Psicología, 104, 37-56.

Depaula, P., Torres, J. A., Piñeyro, D. R., Cosentino, A. C., Clotet, C. I. \& Castillo, S. (2012, Noviembre). Desarrollo de un software para la evaluación de la toma de decisiones en contextos de incertidumbre cultural. Póster presentado en el IV Congreso Internacional de Investigación y Práctica Profesional en Psicología, XIX Jornadas de Investigación, VIII Encuentro de Investigadores en Psicología del MERCOSUR, organizado por la Secretaría de Investigaciones de la Facultad de Psicología de la Universidad de Buenos Aires, Ciudad Autónoma de Buenos Aires, Buenos Aires.

Dreyfus, H. L. \& Dreyfus, S. E. (2005). Expertise in real world contexts. Organization Studies, 26(5), 779-792. doi: 10.1177/0170840605053102

Epstein, S. (1994). Integration of the cognitive and psychodynamic unconscious. American Psychologist, 49, 709-724. doi: 10.1037/0003-066X.49.8.709

Evans, J. St. B. T. (2003). In two minds: dual-process accounts of reasoning. Trends in Cognitive Sciences, 7(10), 454-459. doi: 10.1016/j.tics.2003.08.012

Evans, J. St. B. T. \& Over, D. E. (1996). Rationality and Reasoning. Hove: Psychology Press. 
Fadde, P. J. (2009). Instructional design for advanced learners: Training recognition skills to hasten expertise. Education Tech Research Dev, 57, 359376. doi: 10.1007/s11423-007-9046-5

Frank, G., Curry, R., Wheaton, W., Hill, R. \& Abbott, J. B. (2011, December). Training on Cultural Aspect of Planning Humanitarian Assistance Operations. Paper presented at the Interservice/Industry Training, Simulation, and Education Conference, organized by the National Training and Simulation Association, Orlando, FL.

Gibson, J. J. (1979). The Ecological Approach to Visual Perception. Boston: Houghton Mifflin.

Gigerenzer, G. (2008). Decisiones instintivas. La inteligencia del inconsciente. Barcelona: Ariel.

Gigerenzer, G. (2009). Fast and Frugal Heuristic: The Tools of Bounded Rationality. En D. J. Koehler \& N. Harvey (Eds.), Blackwell Handbook of Judgment \& Decision Making (pp. 62-88). Malden, MA: Blackwell Publishing.

Gigerenzer, G. \& Kurz, E. M. (2001). Vicarious functioning reconsidered: A fast and frugal lens model. En K. R. Hammond \& T. R. Stewart (Eds.), The Essential Brunswik: Beginning, Explications, Applications (pp. 342-347). Oxford: Oxford University Press.

Gigerenzer, G., Todd, P. \& the ABC Research Group. (1999). Simple Heuristics that make Us Smart. New York, NJ: Oxford University Press.

Goldstein, D. G. \& Gigerenzer, G. (2002). Models of Ecological Rationality: The Recognition Heuristic. Psychological Review, 109(1), 75-90. doi: 10.1037//0033-295X.109.1.75

Goldstein, D. G. \& Gigerenzer, G. (2009). Fast and frugal forecasting. International Journal of Forecasting, 25, 760-772. doi: 10.1016/j.ijforecast.2009.05.010

Goldstein, W. M. (2009). Social Judgment Theory: Applying and Extending Brunswik's Probabilistic Functionalism. En: D. J. Koehler, \& N. Harvey (Eds.), Blackwell Handbook of Judgment \& Decision Making (pp. 37-61). Malden, MA: Blackwell Publishing.

Hambrick, D. Z., Altmann, E. M., Oswald, F. L., Meinz, E. J. \& Gobet, F. (2014). Facing facts about deliberate practice. Frontiers in Psychology, 5, 1-2. doi: 10.3389/fpsyg.2014.00751 
Hammond, K. R. (1955). Probabilistic functionalism and the clinical method. Psychological Review, 62, 255-262.

Hammond, K. R. (2010). Intuition, No! ...Quasirationality, Yes! Psychological Inquiry: An International Journal for the Advancement of Psychological Theory, 21(4), 327-337. doi: 10.1080/1047840X.2010.521483

Hammond, K. R. (2015). Causality vs generality: Judgment and decision making struggles to become a scientific discipline. Journal of Applied Research in Memory and Cognition, 4(3), 176-179. doi: 10.1016/j.jarmac.2015.07.002

Harrison, D. A., Shaffer, M. A. \& Bhaskar-Shrinivas, P. (2004). Going places: roads more and less traveled in research on expatriate experiences. Research in Personnel and Human Resources Management, 23, 199-247. doi: 10.1016/ S0742-7301(04)23005-5

Hodgkinson, G. P. \& Starbuck, W. H. (2008). Organizational Decision Making: Mapping Terrains on Different Planets. En G. P. Hodgkinson, \& W. H. Starbuck (Eds.), The Oxford Handbook of Organizational Decision Making (pp. 1-29). Oxford: Oxford University Press.

Hoffrage, U. \& Marewski, J. N. (2015). Unveiling the Lady in Black: Modeling and aiding intuition. Journal of Applied Research in Memory and Cognition, 4, 145-163. doi: 10.1016/j.jarmac.2015.08.001

Hofstede, G. (1980). Culture's consequences: International differences in workrelated values. Newbury Park, CA: Sage.

Hofstede, G. (2001). Culture's consequences: Comparing values, behaviors, institutions, and organizations across nations. Thousand Oaks, CA: Sage.

Horrey, W. J., Lesch, M. F., Mitsopoulos-Rubens, E., \& Lee, J. D. (2015). Calibration of skill and judgment in driving: Development of a conceptual framework and the implications for road safety. Accident Analysis and Prevention, 76, 25-33. doi: 10.1016/j.aap.2014.12.017

Hurbon, L. (1993). Les mystères du Vaudou. París: Gallimard.

Jacobs, J. E. \& Potenza, M. (1991). The use of judgment heuristics to make social and object decisions: A developmental perspective. Child Development, 62, 166-178. doi: 10.1111/j.1467-8624.1991.tb01522.x

Kahneman, D. \& Klein, G. (2009). Conditions for intuitive expertise: A failure to disagree. American Psychologist, 64, 515-526. doi: 10.1037/a0016755 
Klein, G. (1998). Sources of Power: How People Make Decisions. Cambridge, MA: MIT Press.

Klein, G. (2008). Naturalistic Decision Making. Human Factors, 50(3), 456-460. doi: 10.1518/001872008X288385

Laborde, S., Dosseville, F. \& Scelles, N. (2010). Trait emotional intelligence and preference for intuition and deliberation: Respective influence on academic performance. Personality and Individual Differences, 49(7), 784-788. doi: 10.1016/j.paid.2010.06.031

Laborde, S. \& Raab, M. (2013). The Tale of Hearts and Reason: The Influence of mood on Decision Making. Journal of Sport \& Exercise Psychology, 35, 339-357.

Lieberman, M. D., Jarcho, J. M. \& Satpute, A. B. (2004). Evidence-Based and Intuition-Based Self-Knowledge: An fMRI Study. Journal of Personality and Social Psychology, 87(4), 421-435. doi: 10.1037/0022-3514.87.4.421

Lin, Y., Chen, A. S. \& Song, Y. (2012). Does your intelligence help to survive in a foreign jungle? The effects of cultural intelligence and emotional intelligence on cross-cultural adjustment. International Journal of Intercultural Relations, 36, 541- 552. doi: 10.1016/j.jintrel.2012.03.001

Lipshitz, R., Klein, G., Orasanu, J. \& Salas, E. (2001). Focus Article: Taking Stock of Naturalistic Decision Making. Journal of Behavioral Decision Making, 14, 331-352. doi: $10.1002 / \mathrm{bdm} .381$

Macnamara, B. N., Hambrick, D. Z. \& Oswald, F. L. (2014). Deliberate Practice and Performance in Music, Games, Sports, Education, and Professions: A Meta-Analysis. Psychological Science, 1, 1-11. doi: 10.1177/0956797614535810

MacPherson, S. E., Philips, L. H. \& Della Sala, S. (2002). Age, executive function, and social decision making: A dorsolateral prefrontal theory of cognitive aging. Psychology and Aging, 17, 598-609. doi: 10.1037/0882-7974.17.4.598

Markovits, H. \& Dumas, C. (1999). Developmental patterns in the understanding of social and physical transitivity. Journal of Experimental Child Psychology, $73,95-114$.

Marr, D. (1982). Vision. San Francisco: W. H. Freeman.

McKenzie, C. R. M. (2009). Hypothesis Testing and Evaluation. En D. J. Koehler \& N. Harvey (Eds.), Blackwell Handbook of Judgment \& Decision Making (pp. 200-219). Malden, MA: Blackwell Publishing. 
Moxley, J. H., Ericsson, K. A., Charness, N. \& Krampe, R. T. (2012). The role of intuition and deliberative thinking in experts' superior tactical decisionmaking. Cognition, 124, 72-78. doi: 10.1016/j.cognition.2012.03.005

Pacini, R. \& Epstein, S. (1999). The relation of rational and experiential information processing styles to personality, basic beliefs, and the ratio-bias phenomenon. Journal of Personality and Social Psychology, 76, 972-987. doi: 10.1037/0022-3514.76.6.972

Phillips, J. K., Klein, G. \& Winston, R. S. (2009). Expertise in Judgment and Decision Making: A Case for Training Intuitive Decision Skills. En D. J. Koehler, \& N. Harvey (Eds.), Blackwell Handbook of Judgment \& Decision Making (pp. 297-315). Malden, MA: Blackwell Publishing.

Raab, M. (2012). Simple heuristics in sports. International Review of Sport and Exercise Psychology, 5, 104-120. doi: 10.1080/1750984X.2012.654810

Raab, M. \& Laborde, S. (2011). When to Blink and When to Think: Preference for Intuitive Decisions Results in Faster and Better Tactical Choices. Research Quarterly for Exercise and Sport, 82(1), 89-98. doi: 10.5641/027013611X1309 8902481941

Ross, K. G., Phillips, J. K., Klein, G. \& Cohn, J. (2005). Creating expertise: A framework to guide technology-based training. Final Technical Report for Contract \#M67854-04-C-8035 for the Marine Corps Systems Command/ Program Manager for Training Systems. Fairborn, OH: Klein Associates.

Ross, K. G. \& Thornson, C. A. (2008). Toward an operational definition of crosscultural competence from the literature. Patrick AFB, FL: Defense Equal Opportunity Management Institute.

Sadler-Smith, E. (2011). The intuitive style: Relationship with local/global and verbal/visual styles, gender, and superstitious reasoning. Learning and Individual Differences, 21, 263-270. doi: 10.1016/j.lindif.2010.11.013

Salas, E., Rosen, M. A. \& DiazGranados, D. (2010). Expertise-Based Intuition and Decision Making in Organizations. Journal of Management, 4(36), 941973. doi: 10.1177/0149206309350084

Serwe, S. \& Frings, C. (2006). Who will win Wimbledon? The recognition heuristic in predicting sports events. Journal of Behavioral Decision Making, 19(4), 321-332. doi: 10.1002/bdm.530

Simon, H. A. (1955). A Behavioral Model of Rational Choice. Quarterly Journal 
of Economics, 69, 99-118.

Simon, H. A. (1987). Making Management Decisions: The Role of Intuition and Emotion. Academy of Management Executive, 1(1), 57-66.

Sinclair, M. (2010). Misconceptions About Intuition. Psychological Inquiry: An International Journal for the Advancement of Psychological Theory, 21, 378386. doi: 10.1080/1047840X.2010.523874

Sinclair, M. \& Ashkanasy, N. M. (2005). Intuition: Myth or a Decision-making Tool? Management Learning, 36(3), 353-370.doi: 10.1177/1350507605055351

Torres, A., Azzollini, S., Depaula, P. \& Clotet, C. (2010). La incertidumbre del contexto percibida por militares voluntarios en una misión de paz en Haití. En: V. Barilá, A. Lapalma, \& M. J. Molina (Comps.), Libro del XII Congreso Metropolitano de Psicología: psicología y sociedad (pp. 142-144). Buenos Aires: Asociación de Psicólogos de Buenos Aires.

Tulving, E. (1972). Episodic and semantic memory. En E. Tulving, \& W. Donaldson (Eds.), Organization of memory (pp. 37-62). New York, NJ: Academic Press.

Tulving, E. (1983). Elements of episodic memory. Oxford: Clarendon Press.

Wason, P. C. (1968). Reasoning about a rule. Quarterly Journal of Experimental Psychology, 20, 273-281. doi: 10.1080/14640746808400161

Wason, P. C. (1969). Razonamiento. En B. M. Foss (Ed.), Nuevos horizontes en psicología (pp. 132-146). Barcelona: Fontanella.

Zimmerman, L. A., Mueller, S. T., Marcon, J. L., Daniels, J. B. \& Vowels, C. L. (2011). Improving Soldier Threat Detection Skills in the Operational Environment. Interservice/Industry Training, Simulation, and Education Conference. Paper No. 11196 (pp. 1-12). 